\title{
Fièvre hémorragique en Franche-Comté et Hantavirus
}

\section{Hemorrhagic Fever in Franche-Comté and Hantavirus}

\section{A. Sigaux $\cdot$ T. Desmettre}

Reçu le 16 novembre 2017; accepté le 8 janvier 2018

(C) SFMU et Lavoisier SAS 2018

\section{Introduction}

Les Hantavirus appartiennent à la famille Bunyaviridae. Quatre espèces d'Hantavirus zoonotiques sont décrites en Europe. Par ordre décroissant, les virus sont Puumala (PUUV) [présent dans $81 \%$ des pays d'Europe], DobravaBelgrade (37\% des pays d'Europe), Tula (31\% des pays d'Europe) et Séoul (SEOV) [9 \% des pays d'Europe] [1]. Les rongeurs, en particulier le campagnol roussâtre (Clethrionomys glareolus), sont les hôtes naturels de ces virus. La contamination humaine résulte généralement de l'inhalation de poussières, contaminées par les excréments ou la salive des rongeurs. L'homme n'est donc qu'un hôte accidentel de l'infection par Hantavirus. Ces Hantavirus sont responsables d'une fièvre hémorragique avec syndrome rénal (FHSR) [1]. L'évolution des FHSR est le plus souvent favorable, mais des complications sont notées à type de choc hypovolémique, manifestations hémorragiques y compris cérébrales ou encore insuffisance rénale avec anurie. Il n'existe pas de traitement spécifique, et la prise en charge est symptomatique, avec parfois nécessité d'hémodialyse [2]. En France, la majorité des cas sont signalés dans le quart nord-est du pays (Fig. 1) [3]. PUUV est endémique dans cinq zones géographiques : les Ardennes, les environs de Nancy, la Franche-Comté, la vallée de l'Oise et de la Marne [3]. PUUV est responsable d'une forme atténuée de FHSR, causant moins de $0,5 \%$ de décès.

Nous rapportons l'observation en Franche-Comté d'un cas probable d'infection par PUUV, et en détaillons les caractéristiques cliniques, paracliniques et évolutives.

A. Sigaux $(\bowtie) \cdot$ T. Desmettre

Service d'accueil des urgences/Samu 25, université de Bourgogne-Franche-Comté, centre hospitalier universitaire de Besançon, 3, boulevard Fleming, F-25030 Besançon cedex, France e-mail : asigaux@chu-besancon.fr

\section{Cas clinique}

Un homme de 62 ans consultait au service d'accueil des urgences pour une insuffisance rénale aiguë dans un contexte fébrile. Il s'agissait d'un patient actif, sportif et résidant en zone rurale dans le Haut-Jura. Le patient était hypertendu sous antagoniste des récepteurs de l'angiotensine 2 (ARA2). Le patient présentait une altération de l'état général accompagnée de fièvre avec pics fébriles à $39,5^{\circ} \mathrm{C}$ depuis trois jours. Il n'existait pas de douleur. Quelques troubles de l'accommodation étaient rapportés par le patient. Il consulta son médecin traitant la veille de son admission qui prescrivit un bilan sanguin retrouvant une insuffisance rénale aiguë avec créatinine à $348 \mu \mathrm{mol} / \mathrm{l}$, une thrombopénie à $112 \mathrm{G} / \mathrm{l}$ et une cytolyse hépatique avec aspartate aminotransférase à $109 \mathrm{UI} / 1$ et alanine aminotransférase à $145 \mathrm{UI} / 1$. La protéine $\mathrm{C}$ réactive (CRP) s'élevait à $40 \mathrm{mg} / 1$ sans hyperleucocytose. Les sérologies EpsteinBarr, cytomégalovirus, toxoplasmose, Lyme, hépatites A, $\mathrm{B}$ et $\mathrm{C}$ et VIH étaient négatives. Il était adressé au service des urgences. À l'arrivée au service des urgences, la pression artérielle était de $141 / 79 \mathrm{mmHg}$, la fréquence cardiaque était de 80 battements par minute, la saturation en oxygène était de $98 \%$ en air ambiant et la température de $36,4^{\circ} \mathrm{C}$. La bandelette urinaire retrouvait une protéinurie supérieure à $3 \mathrm{~g} / \mathrm{l}$. L'examen physique était sans particularité. Le bilan biologique retrouvait une créatinine à $394 \mu \mathrm{mol} / 1$ avec une hypokaliémie à $3,3 \mathrm{mmol} / \mathrm{l}$. L'urée était à $23 \mathrm{mmol} / \mathrm{l}$. Les valeurs de la cytolyse ainsi que de la CRP étaient stables. La diurèse était conservée. Après interrogatoire orienté, il était noté des travaux de nettoyage d'un bûcher un mois avant la symptomatologie décrite. L'échographie abdominopelvienne était sans particularité. Une hospitalisation de 48 heures en néphrologie a été effectuée. Le résultat du test rapide Reagana Puumala IgM était positif. Avec un traitement symptomatique et une suspension de l'ARA2, l'évolution était favorable en sept jours. Les valeurs de transaminases, de créatininémie et de la formule sanguine s'étaient normalisées. Ce résultat de sérologie Hantavirus a été confirmé par le Centre national de 


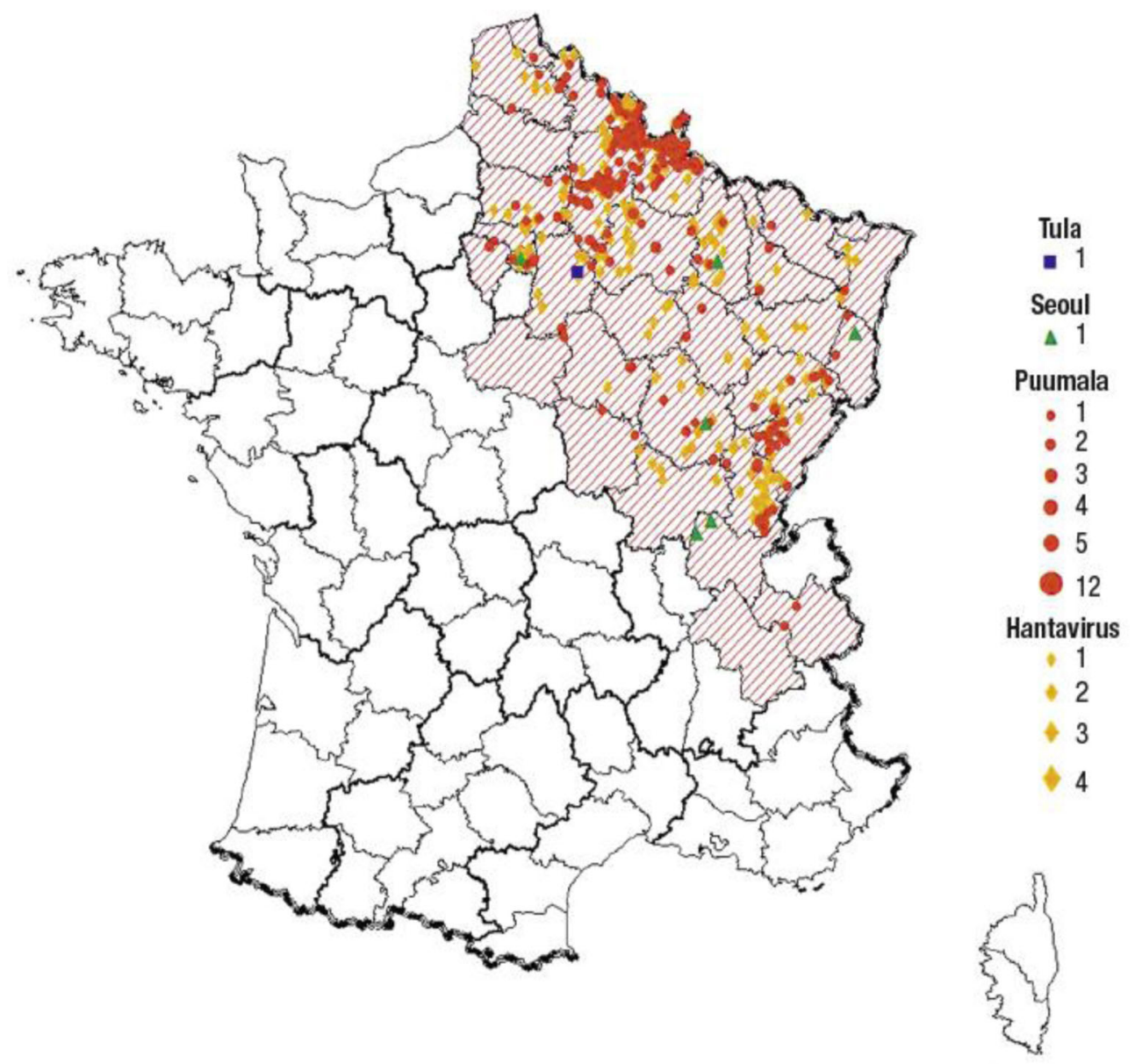

Fig. 1 Distribution spatiale des 470 cas confirmés d'infection récente par un Hantavirus en France métropolitaine de 2012 à 2016 [3]. Distribution établie sur la base de la commune d'exposition ou de la commune de résidence si une commune d'exposition n'est pas indiquée ; en hachuré, figurent les départements où des cas ont été détectés sur la période 2003-2016 (zone d'endémie). « Hantavirus » correspond aux cas confirmés par sérologie seulement et pour lesquels l'espèce virale n'a pu être identifiée. Reproduit d'après Reynes et al. [3] avec autorisation

référence (CNR) des Hantavirus, avec la mise en évidence de PUUV et d'IgM dirigées contre PUUV dans le même échantillon.

\section{Discussion}

Après une incubation d'une à huit semaines [4], les manifestations cliniques sont un syndrome grippal brutal (fébrile : $39-40^{\circ} \mathrm{C}$ initialement, puis apyrexie) pouvant comprendre des douleurs abdominales, articulaires ou vertébrales, des céphalées ainsi qu'une myopie aiguë [5]. Il s'agit d'un tableau peu spécifique, et la confusion avec d'autres diagnostics est fréquente. Une phase hypotensive initiale peut être observée, laissant place ensuite à une phase oligurique et hypertensive durant quelques jours [3]. L'anamnèse soigneuse revêt une importance capitale dans la démarche diagnostique. Dans notre cas, il existait des troubles de l'accom- modation visuelle qui est un signe pathognomonique de l'infection et présente dans un tiers des cas. Elle est bien décrite dans les infections par SEOV et PUUV [6]. Elle peut se manifester par un authentique trouble de la réfraction, ou une simple impression subjective de vision floue, ou des hémorragies conjonctivales [6]. Un autre élément anamnestique majeur était l'activité forestière qui l'exposait aux excréments de rongeurs, notamment dans le cadre du stockage de bois [7]. Les professions à risque sont les travailleurs forestiers, les chasseurs, les militaires [4]. Les activités de loisirs en milieu forestier, la coupe de bois et le nettoyage de greniers sont aussi rapportés comme facteurs de risque [4]. Les examens biologiques mettent en évidence une insuffisance rénale aiguë qui reste l'élément diagnostique principal [8], secondaire à une néphrite interstitielle aiguë [8] avec des suffusions hémorragiques. On note une protéinurie dans $70 \%$ des cas, souvent supérieure $2 \mathrm{~g} / 1$ [7]. Le recours à l'hémodialyse est nécessaire dans moins de $10 \%$ des cas. 
La seconde anomalie biologique caractéristique est une thrombopénie périphérique souvent supérieure à 50000 plaquettes $/ \mathrm{mm}^{3}$, présente dans $98 \%$ des cas [9]. Les manifestations hémorragiques sont rares en dehors d'une hématurie dans $35 \%$ des cas. Cependant, dans de rares cas, il y a apparition de complications sévères, qui peuvent être mortelles, comme l'apparition de symptômes hémorragiques, associés ou non à une insuffisance rénale aiguë, ou de troubles neurologiques (coma...). Toutefois, cette mortalité n'est retrouvée actuellement que dans 0,1 à $0,2 \%$ des cas. Dans le cadre des examens complémentaires, l'échographie peut montrer des aspects typiques de cette infection avec des gros reins hyperréflectifs, des épanchements périrénaux, abdominaux, pleuraux et une splénomégalie. Le traitement de la virose est purement symptomatique avec éviction des néphrotoxiques. La convalescence s'étale de trois semaines à trois mois avec disparition des signes clinicobiologiques. Les séquelles (hypertension artérielle, protéinurie résiduelle, insuffisance rénale chronique) sont exceptionnelles. Le diagnostic est sérologique, les IgM et IgG anti-PUUV apparaissent très rapidement après l'apparition des signes cliniques et sont présentes chez quasiment tous les patients dès le septième jour d'évolution de la maladie.

En France métropolitaine, la surveillance des infections par les Hantavirus est réalisée par le CNR en lien avec les laboratoires qui effectuent le diagnostic de première intention. Celui-ci est réalisé par un test rapide de dépistage des IgM, permettant d'avoir une réponse dans les deux heures. Ces laboratoires adressent au CNR, à des fins de surveillance et de confirmation, des prélèvements présentant un résultat positif mais également ceux avec un résultat limite ou négatif peu compatible avec la situation clinique.

À l'occasion d'un épisode de FHSR, les anticorps persistent longtemps et assurent ainsi une immunité acquise. Des vaccins contre certains virus sont disponibles uniquement dans quelques pays asiatiques. Enfin, l'incidence des infections humaines par les Hantavirus augmente lorsque les conditions climatiques et une nourriture abondante favorisent l'augmentation des populations de son hôte rongeur [1]. En France, cette situation se produit le plus souvent entre mai et août, avec un pic de détection au mois de juin [10]. De 2005 à 2015, 1210 cas ont été rapportés dans le pays, avec une moyenne de 50 à 200 cas/an, dont environ $40 \%$ dans le massif ardennais [3]. Face à une présentation clinique et biologique compatible, il s'avère pertinent d'évoquer le diagnostic d'infection par Hantavirus, quelle que soit la localisation géographique.

\section{Conclusion}

L'infection par Hantavirus est un diagnostic à évoquer sans retard devant l'association d'une fièvre, d'une insuffisance rénale aiguë et d'une thrombopénie. Une élévation des transaminases ne doit pas remettre en cause le diagnostic. Les troubles visuels transitoires sont spécifiques de cette infection et doivent alerter le clinicien. Néanmoins, la FHSR est probablement sous-diagnostiquée en zone épidémique par son mode d'entrée aspécifique. Une recherche systématique sérologique des cas de FHSR permettrait d'éviter de nombreuses explorations inutiles et d'anticiper les conséquences possibles, en particulier rénales.

Conflits d'intérêts : les auteurs déclarent ne pas avoir de conflits d'intérêts.

\section{Références}

1. Olsson GE, Leirs H, Henttonen H (2010) Hantaviruses and their hosts in Europe: reservoirs here and there, but not everywhere? Vector Borne Zoonotic Dis 10:549-61.

2. Avšič Županc T, Korva M, Markotić A (2014) HFRS and Hantaviruses in the Balkans/South-East Europe. Virus Res 187:27-33

3. Reynes JM, Carli D, Renaudin B, et al (2017) Surveillance des infections humaines par Hantavirus en France métropolitaine, 2012-2016. Bull Epidemiol Hebd 23:492-9 http://invs.santepubliquefrance.fr/beh/2017/23/2017_23_1.html (Dernier accès le 14 décembre 2017)

4. Bi Z, Formenty PBH, Roth CE (2008) Hantavirus infection: a review and global update. J Infect Dev Ctries 2:3-23

5. Noh JY, Cheong HJ, Song JY, et al (2013) Clinical and molecular epidemiological features of hemorrhagic fever with renal syndrome in Korea over a 10-year period. J Clin Virol 58:11-7

6. Theiler G, Langer-Wegscheider B, Zollner-Schwetz I, et al (2012) Blurred vision and myopic shift in Puumala virus infections are independent of disease severity. Eur J Clin Microbiol Infect Dis 18:435-437

7. Strady C, Penalba C, Baumard S, et al (2009) Nephropathia epidemica and leptospirosis in Champagne-Ardenne, France: comparison of clinical, biological and epidemiological profiles. Eur J Clin Microbiol Infect Dis 28:825-9

8. Denecke B, Bigalke B, Haap M, et al (2010) Hantavirus infection: a neglected diagnosis in thrombocytopenia and fever? Mayo Clin Proc 85:1016-20

9. Laine O, Joutsi-Korhonen L, Lassila R, et al (2015) Hantavirus infection-induced thrombocytopenia triggers increased production but associates with impaired aggregation of platelets except for collagen. Thromb Res 136:1126-32

10. Belchior E, Zeller H, Nicolau J, et al (2009) La fièvre hémorragique avec syndrome rénal en France métropolitaine de 2002 à 2007 : données du PMSI et du CNR. Bull Epidemiol Hebd http://opac.invs.sante.fr/doc_num.php?explnum_id=1005 (Dernier accès le 11 juillet $201 \overline{7}$ ) 